\title{
The effect of combinations of image degradations in a discrimination task
}

\author{
WILLIAM R. UTTAL, TODD BARUCH, and LINDA ALLEN \\ Arizona State University, Tempe, Arizona
}

\begin{abstract}
This paper explores the ways in which combinations of image degradations affect discrimination. Nine experiments are described that examine the discriminability of visual images that are degraded with three types of information reducing transformations: random punctate visual interference, lowpass spatial frequency filtering, and local area (i.e., block) averaging. The results of these experiments characterize a powerful visual ability to discriminate highly degraded stimuli unless that ability is severely challenged by relatively high levels of random visual interference. Discriminative commutativity of the orders in which the other two degradations are imposed is demonstrated. That is, the order in which the degradations are applied does not affect the final discriminative outcome. This result is in contrast to predictions from relevant mathematics and direct examination of the images produced by both orders of degradation. The commutativity is attributed to the particularly strong effect of the low-pass spatial frequency filtering degradation on the discrimination process. This study also demonstrates that combinations of degradations in a discrimination task always result in a reduction in performance, and never in the improvement that has been reported for recognition. This difference is attributed to the fact that form discrimination is mediated mainly by local features and high-frequency spatial components, whereas recognition is mediated mainly by global features and low-frequency spatial components.
\end{abstract}

The goal of the research reported here is to determine how combining different kinds of image degradations affects the visual processing of stimulus images. Visual images can be degraded in many different ways. Among the most salient to our understanding of human perception are (1) The reduction in signal-to-noise ratios produced by the addition of punctate visual interference (randomly illuminated dots); (2) the changes in images, including a reduction in resolution, that may be caused, for example, by averaging over local areas (blocking); and (3) the blurring and contrast changes that may be caused, for example, by low-pass filtering in which high spatial frequencies are removed from the image.

There is a rich scientific history of studies in which a single degradation was applied to determine the effect on some aspect of visual perception. It would be impossible to review all of them in a brief paper, but it is important to appreciate that band-pass filtering effects on vision have been studied for many years, beginning with the pioneering work of Campbell, Howell, and Robson (1971), Campbell, Howell, and Johnstone (1978), Ginsburg (1978), and Ginsburg and Evans (1979). Full discussions of the impact of spatial frequency filtering on psycho-

This research was supported by Grant F49620-92-J-0176-P00001 from the United States Air Force Office of Scientific Research. The advice of Takeo Watanabe in the preparation of this article is gratefully acknowledged. Correspondence should be addressed to W. R. Uttal, Department of Industrial and Management Systems Engineering, Arizona State University, Tempe, AZ 85287-5906 (e-mail: aowru@ asuvm.inre.asu.edu). physical data can be found in the books by Graham (1989) and by De Valois and De Valois (1988).

The influence of random punctate visual interference also has been extensively studied. Our own work (Uttal, $1975,1983,1985,1987)$ is but a sample of the many studies of the effects of this form of degradation.

The use of blocking as a parametrically controlled stimulus degradation in isolation is rarer. The work of Bachmann is unusual in this regard. In the first of two reports (Bachmann, 1987) on this topic, he varied block size and found quantitatively and qualitatively different patterns of response as a function of this variable. In a second report (Bachmann, 1991), he showed that there was a discontinuity in recognizability when the block size was increased from 15 to 18 blocks across a face even though all smaller blocks produced essentially similar recognition scores.

Blocking has also been used occasionally in the study of cascaded or combined sequential degradations, particularly in studies of recognition. The latter approach was pioneered in the now classic study reported by Harmon and Julesz (1973), in which it was asserted that subsequent low-pass filtering of a blocked image improved recognition. They attributed the phenomenon of improved recognition to the removal of specific bands of high-frequency components (the edges introduced by the blocking degradation) by the low-pass filter. Their explanatory conjecture was that critical bands of highfrequency spatial components of the blocked structure masked the low-frequency components carrying the information necessary for recognition. 
Harmon and Julesz's (1973) demonstration of the phenomenon has stimulated a considerable amount of research since that original publication. However, although the reality of the phenomenon is generally accepted, not everyone has concurred with their conjecture that the diminishment in performance following blocking was due to high-frequency masking. One report that supports this hypothesis (Parker \& Costen, 1993) describes a nonlinear increase in the decline in recognizability as the block size increases. They interpret this nonlinearity to be evidence for a critical band of spatial frequencies. Morrone, Burr, and Ross (1983), on the other hand, added noise in what was the critical high-frequency band and increased recognizability. Because of this result, they rejected the high-frequency masking conjecture, even though accepting the reality of the phenomenon itself. Similarly, Durgin and Proffit (1993) have shown that adding high-frequency energy in the form of fine lines placed at the exact edges of the blocks also improves recognition. This result also challenges Harmon and Julesz's explanation that the high-frequency edges introduced by blocking reduce recognition by a masking process. The conjecture is further challenged by a fact that Morrone et al. (1983) call to our attention - that the energy of the high-frequency edges introduced by blocking is quite low in comparison with that of the lowfrequency components of the image.

Before we set forth the protocol and results of the present study, let us lay to rest one important question-Is the Harmon and Julesz phenomenon (not the explanatory conjecture) generalizable to other stimulus types when recognition is the task? In particular, does it hold for objects that are originally silhouettes as well as for gray-scale faces? This question can be answered affirmatively. In the follow-up (Uttal, Baruch, \& Allen, 1995) to this article, we report that the improvement observed in a recognition paradigm occurs with the same type of silhouette stimuli as that used in the present study. This occurs when the stimuli are sequentially blocked and then low-pass filtered, as originally reported for face stimuli. The present results for a discrimination task, therefore, do not dispute the Harmon and Julesz phenomenon in a recognition mode even when tested on a type of stimuli other than faces. They do attest, however, to the fact that the phenomenon is task specific. ${ }^{1}$

We now present results that show that the Harmon and Julesz phenomenon does not manifest itself in a discrimination task. These results, in contrast to the recognition study outcome, suggest that different modes of visual information processing are used in discrimination and recognition tasks, respectively, and that the effects are not solely determined by the energy characteristics of the stimuli.

Several empirical issues emerge from our interest in determining the perceptual effects of sequentially applied image degradations. First, what systematic changes occur in discrimination as the various parameters are manipulated? Second, what is the effect of permuting the order in which the degradations are applied? Third, does the human psychophysical data match predictions from relevant mathematical analyses? Fourth, how can we explain the discrepancy between the results for the recognition and discrimination tasks?

The question of the order of degradations is particularly important. Order effects are significant in establishing the salience of alternative theoretical explanations of the paradoxical effects first reported for recognition. For example, since the outcome of this study differs from that obtained in a recognition task, additional questions arise concerning the relevance of the energy-determined, low-level, spatial frequency based model offered by Harmon and Julesz (1973).

\section{METHOD}

\section{Subjects}

Between 5 and 8 undergraduate students, all with normal or corrected vision, participated as subjects in each of the experiments reported in this article. All were paid an hourly stipend plus a bonus for completion of 3 months of participation. All, therefore, were well-trained observers who stayed in the study for at least several of the experiments. However, because of the length of the study, different groups of individuals participated in different experiments over the course of the study. The subjects were pretrained for at least three sessions before they participated in any of the experiments reported here.

\section{Stimulus Materials}

The psychophysical task used throughout all of the experiments in this study requires the subject to discriminate between the two sequentially presented stimuli transformed by variable degrees and combinations of the three kinds of image degradations - random visual interference, blocking, and low-pass filtering. The stimulus forms to be discriminated were selected from a library of 12 solid silhouettes of aircraft (shown in Figure 1) captured into the computer memory from photographic materials by using a conventional image-capturing video camera. Each of the silhouettes was aligned so that the aircraft were pointing toward the 12:00 position. The silhouettes were always presented singly and placed at the center of the region defined by the punctate visual interference in each presentation of a trial. The silhouettes all subtended approximately $1^{\circ}$ vertically.

The number of aircraft silhouette stimuli used varied from experiment to experiment. In Experiment 1, all 12 shown in Figure 1 were used. In Experiment 3, 6 (2, 4, 5, 6, 10, and 12 from Figure 1) were used; and in all other experiments, $4(2,6,11$, and 12 from Figure 1) were used. This was primarily a practical matter - the preparation time for the many different stimulus conditions increased drastically as we proceeded through the experimental series. Since the response was the outcome of a two-alternative procedure, to a first approximation 50\% was the base level for totally indiscriminate behavior. However, a second approximation suggests that if subjects adopted some response maximizing strategy, a larger number of alternatives could increase performance in complex ways. In fact, most of our data decline to a $50 \%$ base.

Silhouette stimuli are acceptable stimuli in this type of experiment for several reasons. Edges play an important role in recognition and discrimination tasks. The works of Marr (1982) and Shepherd, Uttal, Dayanand, and Lovell (1992) support the notion of boundaries and contours as important determinants of visual perception. It is also important to determine whether the Harmon and Julesz phenomenon generalizes to other types of stimuli. (As noted above, it does so in a surprising way-in a recognition task-but not, as we shall shortly see, in a discrimination task.) 


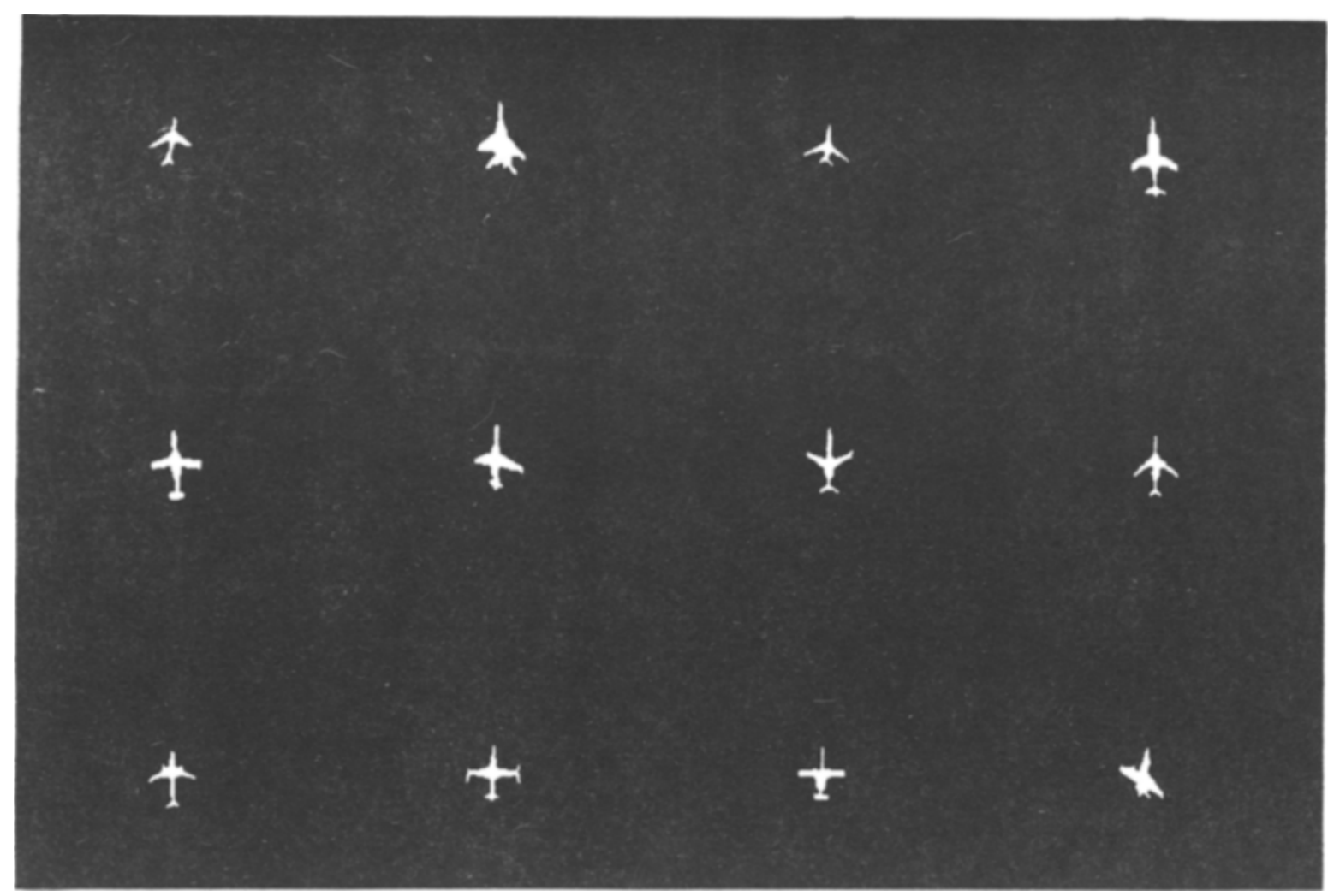

Figure 1. The 12 aircraft silhouettes from which stimuli were chosen for use in the experiments reported in this article. Top row, from left to right: Silhouettes 1-4; middle row, Silhouettes 5-8; bottom row, Silhouettes 9-12.

Furthermore, silhouettes are only initially different from grayscale images. Blocking and spatial frequency filtering both introduce nonhomogenities into what had originally been the undegraded binary images: As block size becomes larger and the cutoff frequencies of a spatial filter become lower, there is more and more internal structure (within the boundaries of what had originally been a homogeneous region - the interior of the silhouette) and the stimuli become gray-scale images.

It must also be pointed out that the nature of the stimulus is always a critical variable in form perception experiments. A test of the generality of any theory with different stimuli is, therefore, desirable. We are currently carrying out both recognition and discrimination studies with faces to supplement the present report.

A typical presentation frame (one of two scquentially presented in the same position on the face of the computer display in each trial) is shown in Figure 2. As we shall see, subjects do amazingly well at discriminating these badly degraded stimuli.

\section{Stimulus Degradations}

Stimuli to be discriminated in each trial were transformed and degraded by applying the combinations of the three degrading transformations shown in Table 1. Stimuli are degraded by applying the "averaging over a region" algorithm (blocking), by filtering the image by removing spatial frequencies higher than a specified cutoff frequency from the spatial frequency spectrum of the image (filtering), or by embedding the stimulus in randomly illuminated pixels (visual interference).

The punctate visual interference used in this experiment was intended to make the task difficult enough so that the effect of the other two degradations would be measurable. Without the chal- lenge to the visual system provided by the interference, discrimination was simply too good to determine subtle effects.

Nine experiments were carried out using the three forms of degradation in various combinations singly to establish baseline levels (Experiments 1, 2, and 4), in pairs (Experiments 3, 5, 6, and 7 ), or in triples (Experiments 8 and 9) to evaluate the combined ef-

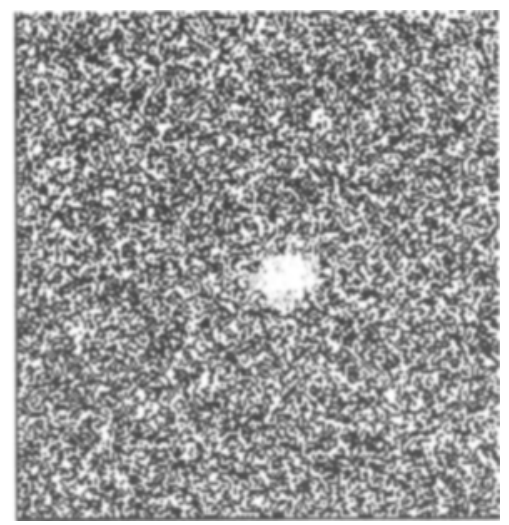

Figure 2. A typical sample of one of two stimulus presentations to be discriminated. This particular stimulus consists of an aircraft silhouette that has been blocked by averaging over a $7 \times 7$ square pixel region and then low-pass spatial frequency filtered with a nominal upper band-pass limit of 1.31 cycles/deg. The filtered stimulus is em- 
Table 1

\begin{tabular}{clll}
\hline Experiment & & & \\
\hline 1 & V.I. & & \\
2 & Filtering & V.I. \\
3 & Filtering & & \\
4 & Blocking & V.I. & \\
5 & Blocking & Filtering & B.I. \\
6 & Blocking & Blocking & V.I. \\
7 & Filtering & Filtering & Blocking \\
8 & Blocking & & \\
9 & Filtering & .
\end{tabular}

V.I. $=$ Visual interference

fects of the degradations on discrimination performance. In Experiments $3,5,8$, and 9 degraded stimuli were embedded in various levels of visual interference. The order of combination of spatial frequency filtering and blocking was the main variable of interest in Experiments 6, 7, 8, and 9. Table 1 summarizes the conditions used in the nine experiments and the overall design of this study.

Low-pass spatial frequency filter. The low-pass filter degradation is accomplished by transforming the aircraft silhouette from the image domain $(x, y)$ to the frequency domain $(u, v)$ by applying a discrete fast Fourier transform (FFT). In principle, a low-pass limit (the cutoff frequency) would then be imposed on the frequencies that are used by a discrete inverse FFT (IFFT) to transform the frequency domain representation back to the image domain. By excluding frequencies higher than certain values (measured in terms of cycles/degree of visual angle on the display) from the IFFT, variable degrees of blurring can be imposed upon the original image.

However, in practice, the use of a simple, sharp cutoff frequency creates a situation that is both unrealistic and problematical. It is unrealistic, because electronic devices do not have sharp cutoff frequencies, but gradual declines. It is problematical, because the use of such an ideal, sharp cutoff in an IFFT produces ringing or oscillation in the inversely transformed image. Ringing could produce confounding distortions in the display, particularly with stimuli of the kind used in this study.

Therefore, rather than employing an ideal sharp cutoff filter to limit the range of frequencies from the frequency domain space, we chose to use a symmetrical (in $u$ and $v$ ), first-order, Butterworth filter. The Butterworth filter's function can be represented by the following expression:

$$
H=\frac{1}{1+(\sqrt{2}-1) x\left(\frac{\sqrt{u^{2}+v^{2}}}{f_{c o}}\right)^{2}}
$$

where $u$ and $v$ are the coordinates of the spatial frequency being attenuated, $H$ is the correction factor (i.e., the value of the Butterworth filter) applied at the spatial frequency $u, v$, and $f_{\mathrm{co}}$ is the nominal cutoff frequency. The Butterworth filter is adjusted so that the attenuation is equal to $1 / \sqrt{2}$ at $f_{\mathrm{co}}$. This produces a gradual dropoff in the amplitude of the frequency components rather than the abrupt discontinuity that would have been introduced by a sharp cutoff limit. Butterworth filtering passes somewhat more energy at frequencies greater than $f_{\mathrm{co}}$ and somewhat less at frequencies less than $f_{\text {co }}$ than a sharp cutoff filter would have. Though this makes our experiments somewhat different from others in which sharp cutoff filtering has been used, it does remove a major potential artifact from our experimental design. It must be remembered, however, that the specified cutoff limits are only nominal. The actual "cutoff" is gradual and precisely defined by Equation 1.

The entire filtering operation is carried out on an automated system that graphically designates the spatial frequency regions that are removed prior to the inverse transformation. ${ }^{2}$ The results of applying the Fourier transform at several different high-frequency nominal cutoff values to one of our sample stimuli is shown in Figure $3 \mathrm{~A}$.

Area averaging (blocking). The second type of image degradation we use is similar, but not identical, to the sample and quantize

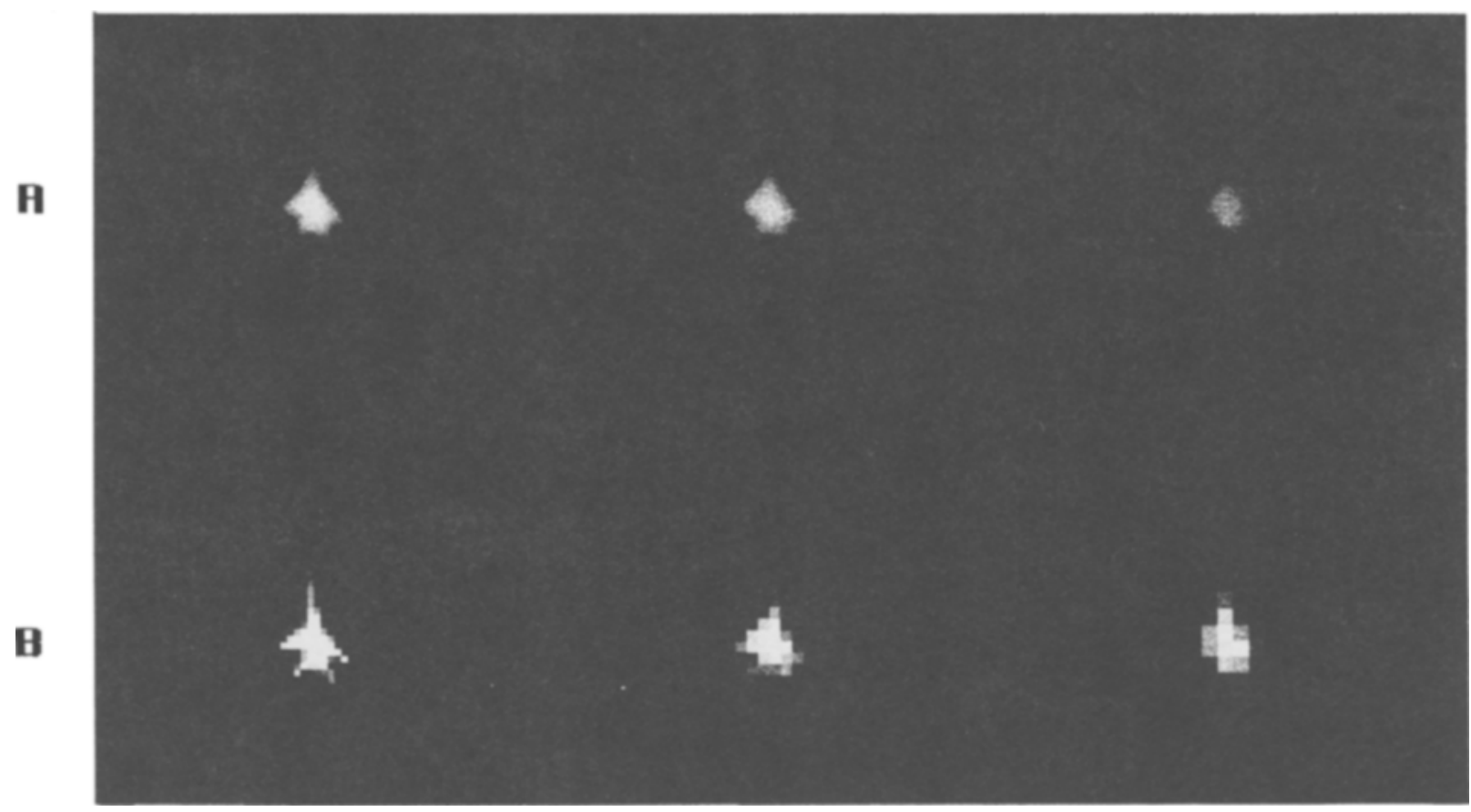

Figure 3. (A) These three sample stimuli show the effects of band-pass spatial frequency filtering with progressively lower limits. From left to right, the nominal upper limits were $1.31, .96$, and .61 cycles/deg, respectively. (B) These three stimuli show the effects of blocking the stimuli by averaging over local regions of $3 \times 3,5 \times 5$, and $7 \times 7$ pixels, respectively. 
technique used in the original Harmon and Julesz (1973) study. In our study, all of the pixel intensity values within a square region of variable size are averaged. The average intensity value is then assigned to all of the pixels in that region. Varying the size of the region degrades the resolution of the image in a controlled manner. The visual angle of a pixel is defined by the width of the viewing region (in degrees of visual angle) divided by the number of pixels across the viewing regions. For our viewing region (defined by the extent of the visual interference), these values are $4.49^{\circ}$ and 200 pixels, respectively. Therefore, a single mathematically defined pixel subtends approximately $1.34^{\prime}$ of visual angle. Averaging was carried out over an $n$ pixel $\times n$ pixel region. The size of the averaging regions will be specified by $n$ in subsequent discussions of the specific experimental conditions. Unlike the Harmon and Julesz technique, ours did not constrain the obtained average pixel intensity values to any particular quantal values. Whatever average value was computed was used as the value of all of the pixels in the averaging region.

The effect of this transformation is to reduce the resolution of the image. Figure 3B shows the results of applying the blocking algorithm to one of our stimuli for several different averaging region sizes.

Random punctate visual interference. Random visual interference was not a form of degradation of primary importance to us in this study. Its function was only to make the task difficult enough to produce differential performance as one or the other (or both) of the other two degradations was varied. The challenge provided by the random visual interference overcame the powerful visual capabilities of the human observer to read through spatial frequency filtering or blocking degradations.

Punctate noise of the kind that we use here is not the same as the band-pass filtered visual "noise" used in some previous experiments. A plot of its spectrum shows that it contains nearly all frequencies (as we demonstrated by applying the FFT transform to a sample of these small dots) and thus represents a very broad band kind of interference. The physical size of each dot was essentially determined by the point spread function of the display. The perceptually significant size of each dot was further modulated by the point spread function of the human eye. Furthermore, the dots of the visual interference were not blocked or spatial frequency filtered as were the stimulus silhouettes.

The density of the visual interference is defined as the proportion of illuminated pixels (i.e., pixels set to the fully illuminated level) for each condition of this study. The visual interference was generated by applying a probabilistic rule that individually determined whether or not a white dot was placed in each of the $200^{2}$ pixel locations within the $4.49^{\circ} \times 4.49^{\circ}$ viewing region on the display screen. The higher the probability, the more the illuminated pixels that were present and the more difficult it was to discriminate the stimulus. As the number of illuminated pixels increases, of course, the overall luminance of the screen increases. However, we have shown in a previous paper (Uttal, Baruch, \& Allen, 1994) that screen illumination and adaptation level have a vanishing small effect in form perception tasks of the kind used here. We extrapolate from those results to the conclusion that stimulus intensity is not a significant variable in the experiments reported here. Of course, contrast is significant and does vary with both filtering and blocking.

The visual interference was applied to the entire viewing region, including the background and the aircraft silhouette, which may have been degraded by neither, one, or both of the other two degradations. If an interference dot was present, its value (full illumination of that pixel) was assigned to that location. If not, either the gray level value of the degraded image or the black of the background was used as the value of that pixel location. This is equivalent to determining the maximum value of the interference or the image at each point, and it places interference dots on both the stimulus form and the background. This operation, therefore, has the property of substituting fully illuminated interference dots for the pixels that correspond to randomly chosen locations without affecting any gray-scale values for pixels at other locations.

The role of the visual interference is especially important in our experiments. As their density increases, the randomly illuminated pixels of the visual interference tend to make the discrimination task increasingly difficult. Indeed, the degrading effect of the random interference is sometimes necessary to unleash any measurable perceptual effect of the other variables. Finally, it is important to note that exactly the same pattern of visual interference is used in both of the stimuli in this discrimination paradigm.

\section{Apparatus}

The experiments reported in this article were carried out on Apollo 3000 computer graphic workstations. Observers were seated with their heads constrained by a chinrest so that their eyes were $64.7 \mathrm{~cm}$ from the face of the display. The entire experimental procedure was controlled by a computer program that randomly selected the stimuli, prepared and presented the stimulus sequence for each trial, collected the observer's responses (by noting which button on the computer mouse was depressed), and then performed a preliminary analysis of the data obtained in each hour-long session.

The viewing region defined by the visual interference was $5.08 \mathrm{~cm}$ $\times 5.08 \mathrm{~cm}\left(4.49^{\circ} \times 4.49^{\circ}\right.$ of visual angle $)$ in extent. Individual pixels appeared as well-focused small white dots when illuminated in isolation. The display itself was the standard raster scan, 16-in. CRT (full screen size was $1,024 \times 768$ pixels) with the relatively long phosphor persistence typically used on workstations. Thus, the duration of the stimulus was considerably longer than the nominal values set in the control program. Since this experiment was not intended to explore any parameter of visual duration or timing, and because the duration of the stimulus was constant throughout the experiment, this persistence introduces no difficulty into our design.

The apparent persistence of the phosphor glow was, however, substantially reduced by indirectly lit incandescent room illumination of approximately $1 \mathrm{~cd} / \mathrm{m}^{2}$ (measured by determining the amount of light reflected from a sheet of white paper at the surface of the display with a Tektronix J17 photometer with a J1803 head) Veiling (as exemplified by its use by Groner, Groner, Muller, Bischof, \& Di Lollo, 1993) is a well-established technique for reducing the effective persistence of an oscilloscopic display. The process depends on the reduction of the contrast of the dimmer temporal "tail" of the phosphor display in comparison with the brighter early portions. The ambient veiling light also provided a constant lighting environment that stabilized the subject's adaptation level and fixation between trials.

As a general calibration procedure, the luminance of a test pattern consisting of the fully illuminated screen (i.e., all pixels set to white) was adjusted each day to $36 \mathrm{~cd} / \mathrm{m}^{2}$ with the Tektronix photometer. This was done with the veiling light present.

\section{General Procedure}

The experimental procedure used in this study was fully automated. Subjects signed into each session by typing their names on the computer keyboard. This initiated a sequence of actions in which the experiment assigned for that session was loaded and the computer was configured to present the appropriate stimuli.

The subjects were instructed to specify whether the two sequentially presented stimuli in each experimental trial were the same or different. A trial consisted of a sequence of visual displays on the CRT. A fixation point was first presented at the center of the display. This was followed by a 500-msec blank period. The first stimulus display was then presented for $100 \mathrm{msec}$ (plus the combined persistence time of the display and the subject's visual system). A blank screen was then presented for $1,025 \mathrm{msec}$. It was followed by the second stimulus display, the temporal duration of 
which was the same as that of the first. Following another 500msec blank period, a question mark appeared on the display that instructed the subject to respond by pushing the left mouse button to indicate "same" or the right button to indicate "different." As soon as the subject responded, a "+" or a "-" sign appeared, which indicated whether the discrimination had been correct or incorrect. The fixation point for the next trial was then displayed and the cycle repeated.

Because of the automated nature of our laboratory and the rapid exchange of stimulus materials between trials, large amounts of data (approximately 200 trials) were collected in each session. The data obtained from all participating subjects were pooled across days to provide the final values plotted on our figures. Within each experiment, all conditions were presented each day to balance out any possible sequence effects. The stimulus combinations presented in each trial were determined by random selection with replacement. The one constraint imposed on stimulus selection was that the number of trials in which the two stimuli were the same was equal to the number of trials in which they differed. We also included appropriate control conditions in each experiment to serve as anchors and references to data from the other experiments.

The particular stimuli used are listed in the description of each experiment. The key idea is that two sequentially presented stimuli to be discriminated are degraded by exactly the same combinations of the three forms of degradation. This included the random visual interference; exactly the same dot pattern was used for both presentations.

\section{EXPERIMENTS AND RESULTS}

\section{Experiment 1: Calibration of Visual Interference}

In the first experiment, the effect of the visual interference was calibrated in the absence of either of the other two forms of degradation-spatial frequency filtering or blocking. Subjects were required, in each trial, to discriminate between two sequential stimuli embedded in the same density and pattern of visual interference. Seven interference levels, ranging from $20 \%$ to $80 \%$ in increments of $10 \%$, were used as independent variable values. All interference values were presented in random order each day. All 12 of the aircraft silhouettes shown in Figure 1 were used in this initial experiment. Eight subjects participated in this experiment for seven daily sessions of $45 \mathrm{~min}$.

The results of this initial calibration experiment are shown in Figure 4. In this and in all subsequent experiments, the plotted points represent the mean of the percent correct scores for all subjects for all of the sessions for that experiment. Since each daily session consisted of approximately 200 trials, and 8 subjects participated in seven sessions, each point plotted on this figure represents a mean score based on 1,600 trials. The vertical lines attached to each data point represent \pm one standard error in this and all subsequent figures.

The general trend of the resulting curve is an accelerating, monotonic decline in the performance scores with increases in the density of the visual interference. That is, a decrease in correct performance of only $10 \%$ occurs between the $20 \%$ and $50 \%$ levels of visual interference, whereas a similar decrease of $10 \%$ occurs between the $50 \%$ and $70 \%$ levels of visual interference. Finally, a $16 \%$ decline in performance occurs for the last $10 \%$ between $70 \%$ and $80 \%$.

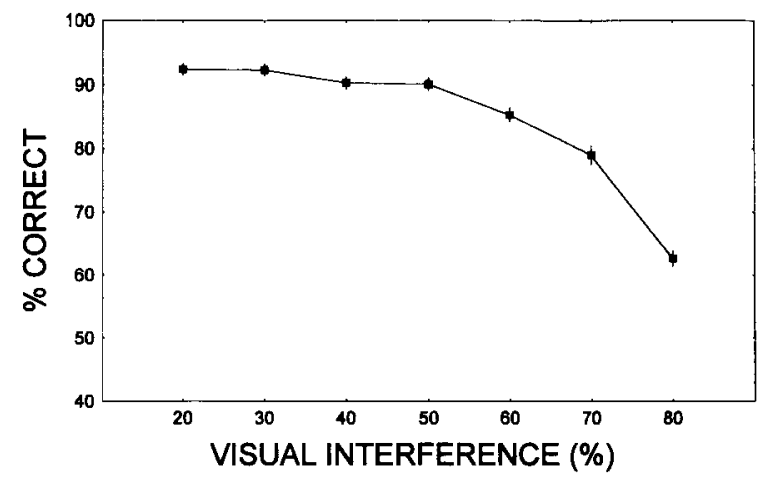

Figure 4. The results of Experiment 1, in which the effects of the density of the visual interference on discrimination performance were measured. In this and all subsequent figures, the "\% Correct" vertical axis designates the average scores for both the trials in which the subjects reported that the stimuli were different when they were different and the trials in which they reported that the stimuli were the same when they were the same. Vertical lines attached to each data point indicate \pm one standard error in this and all subsequent figures. If the vertical lines are absent, the standard error is less than the size of the symbol.

This experiment was necessary in order to help us select useful levels of visual interference with which to test the impact of the other two degradations in Experiments $3,5,8$, and 9 .

\section{Experiment 2: Calibration of the Effect of Low-Pass Filters}

The purpose of the second experiment was to examine the effect on discriminability when the stimuli were degraded solely by low-pass filtering. In this experiment, four of the aircraft silhouettes shown in Figure 1 (Silhouettes 2, 6, 11, and 12) were used. Each of these silhouettes was filtered so that successively broader bands of high-frequency spatial components were removed in four equal steps. That is, the components above nominal cutoff values of $1.13, .96, .78$, and $.61 \mathrm{cycles} / \mathrm{deg}$ of visual angle were removed.

Though these spatial frequency cutoff limits may initially seem rather low, they are in the range for recognition experiments reported by Ginsburg (1980), and they are not dissimilar to the values reported by Hayes, Morrone, and Burr (1986) for at least one of their two viewing distances in a face recognition experiment.

The results of this experiment are shown in Figure 5. Seven subjects each participated in five daily sessions. Therefore, each point on this figure represents the mean obtained from 1,750 trials. The results indicate that the lower the cutoff frequency of the low-pass filter, the lower the performance score. However, the effect of lowpass filtering alone on discrimination performance was relatively modest in comparison with the effects of visual interference. Scores decline from a maximum of about $90 \%$ to a minimum of only $83 \%$. Subjects were able to discriminate relatively well between stimuli that were degraded in the specified manner. 


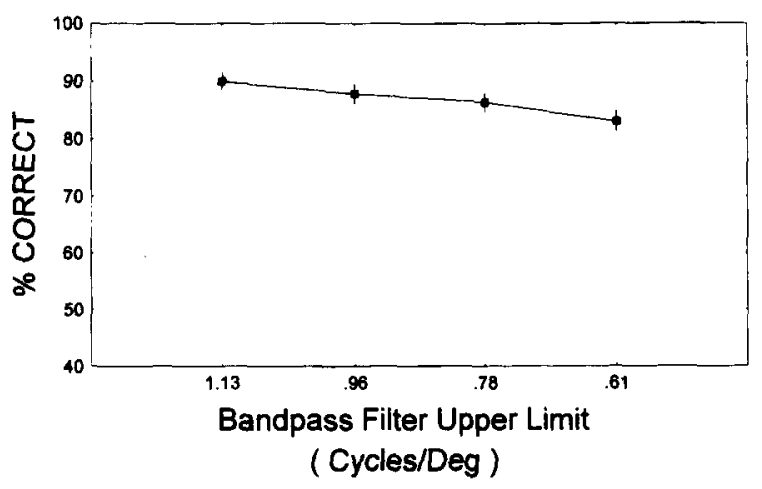

Figure 5. The results of Experiment 2, in which the effect of reducing the nominal upper limit of the spatial frequency filter was measured in the absence of any visual interference.

The significance of these data is that low-pass filtering, in isolation, has a relatively small effect on the discriminability of this type of stimuli. There was sufficient residual form information present to permit high levels of discrimination between the blobs to which these stimuli were degraded. It was, therefore, necessary to degrade the stimuli further, in order to produce a usable level of diminished performance. This led us to the next experiment.

\section{Experiment 3: Combined Effect of Low-Pass Filter and Visual Interference}

Experiment 3 was the first in which two of the three different kinds of degradation were combined. In this experiment, we used six stimuli (Silhouettes 2, 4, 5, 6, 10, and 12 from Figure 1). These stimuli were low-pass filtered as in Experiment 2.

The second kind of degradation used in this experiment was visual interference. Densities of $60 \%, 70 \%$, and $75 \%$ were included in the experimental design. That is, all combinations of stimulus, low-pass filtering, and these three interference levels were presented in random order. Control conditions were also added in which the stimuli were not degraded by the low-pass filter or by the visual interference.

The results of this third experiment are shown in Figure 6. (The data from Experiment 2 have also been replotted in this figure for ease of comparison.) Since 6 subjects were used for five daily sessions, each point on this figure represents the mean performance score obtained from 400 trials.

The performance scores from the noncontrol conditions plotted in this figure display a much greater reduction in discriminability as a result of the simultaneous application of low-pass filtering and visual interference than when either degradation was used alone.

\section{Experiment 4: Calibration of Blocking}

Experiment 4 was the initial calibration study of the effects of blocking (averaging over regions of various sizes) on discriminability. Four stimuli (Silhouettes 2, 6,
11, and 12 from Figure 1) were used in this experiment. These four were chosen because they were roughly of the same size and generally of the same configuration as each other. (As the experimental design became more complex, some parameters of each experiment had to be constrained in order to avoid practical problems associated with the multiplication of conditions.) As described earlier, the blocking procedure averaged pixel intensity values over square (i.e., $n \times n$ pixels) regions. In this experiment, the width and height of the averaging regions in pixels (and minutes of visual angle) used in this experiment were $2\left(2.68^{\prime}\right), 3\left(4.02^{\prime}\right), 4\left(5.36^{\prime}\right), 5\left(6.70^{\prime}\right), 6$ $\left(8.04^{\prime}\right), 7\left(9.38^{\prime}\right)$, and $8\left(10.72^{\prime}\right)$, respectively. A control condition in which there was no blocking of the stimuli (i.e., averaging was carried out over a $1 \times 1$ pixel region) was also included in the design of the experiment.

The results of Experiment 4 are shown in Figure 7. Since each of 5 subjects participated for three sessions, each point on this figure is the mean performance score based on approximately 430 trials. The results of this experiment are notable for demonstrating that there is but

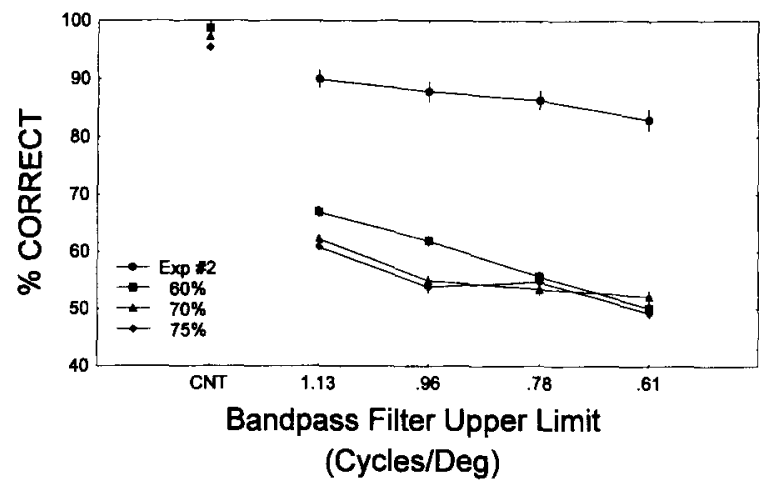

Figure 6. The results of Experiment 3, in which the effect of reducing the nominal upper limit of the spatial frequency filter was measured in three densities of visual interference. The results of $\mathbf{E x}$ periment 2 have also been replotted on this graph for ease of comparison. CNT designates the control stimuli in this and all subsequent figures.

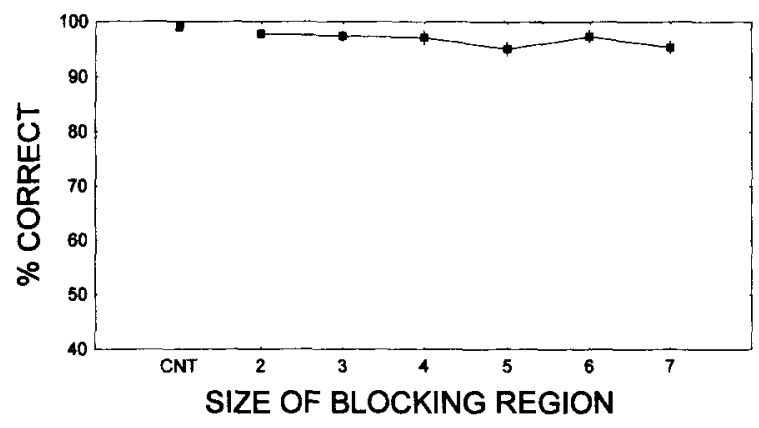

Figure 7. The results of Experiment 4, in which the effect of increasing the size of the averaging area on discrimination was measured in the absence of any visual interference. The horizontal axis refers to the width (and height) of the square region of pixels that were averaged to produce the blocking effect. 
a minimal effect of blocking on visual discrimination under the unchallenged (i.e., no visual interference) conditions of this experiment. In sum, blocked stimuli, undegraded by any additional visual interference, were as discriminable as unblocked ones. This is an expected outcome, given the fact that they, too, were well-defined stimuli with sharp edges (between the averaging regions). Thus, in this case, subjects were merely discriminating forms that, while they may have originally been aircraft silhouettes, were transformed into different "blocky" forms that were also discriminable from each other.

\section{Experiment 5: Combined Effect of Blocking and Visual Interference}

In Experiment 5, we carried the idea of combining degradations to the next step by simultaneously degrading the stimuli with the blocking technique and the random visual interference. Visual interference levels of $60 \%, 70 \%$, and $75 \%$ were again a parameter in this study. The levels of blocking were the same as in Experiment 4, and the same four aircraft silhouette stimuli were used. A control condition was used in which the stimuli were not blocked by local region averaging, only by interference.

Figure 8 shows the results of this experiment. Since 7 subjects participated in this experiment for five sessions each, each point in this figure is the mean performance score based on approximately 550 trials. Data from Experiment 4 have also been replotted on this figure for ease of comparison. In this case, the results are quite different from those of Experiment 4 . Challenged by the random visual interference, performance was much more strongly determined by the size of the region over which pixel intensity values were averaged than in the absence of the visual interference. Whereas Experiment 4 showed only a minor effect of blocking over the range of averaging intervals used, we now observe ranges of performance scores that vary from $14 \%$ to $17 \%$ for each of the three interference densities used. Once again, as in Experiment 3, the curves for the three conditions in which visual interference was used are ap-

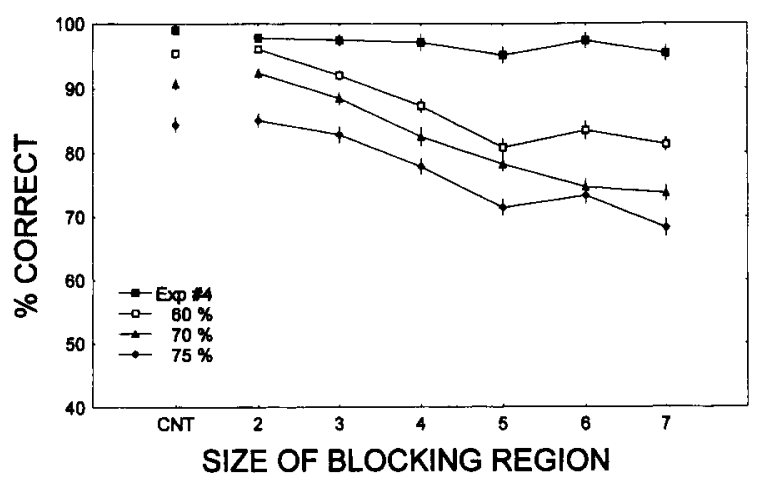

Figure 8. The results of Experiment 5, in which the effect of increasing the size of the averaging region was measured in three different densities of visual interference. The results of Experiment 4 have also been replotted in this figure for ease of comparison.

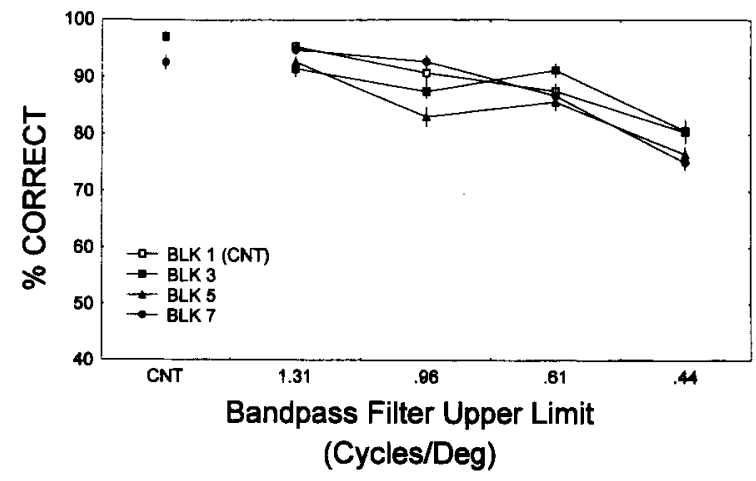

Figure 9. The results of Experiment 6, in which the blocking and the spatial frequency filtering experiments were carried out in sequential order (blocking first, then filtering). The four curves are for four square averaging regions designated by the number of pixels on a side. The four controls in this figure and in Figure 10 are for blocked but unfiltered stimuli. (The control data points for Block Sizes 3, 5 , and 1 are actually overlapping and cannot be plotted separately.)

proximately parallel and exhibit essentially the same slope. Clearly, the addition of punctate visual interference resulted in a substantial decrement in performance.

\section{Experiment 6: Combined Effect of Blocking and Low-Pass Filter}

In Experiment 6, we carried the experimental design of this study to the next step in the sequence of degradation combinations. In this experiment, we tested the subject's discriminative abilities by combining the blocking and the spatial frequency filtering techniques. Blocking was carried out first. The blocked stimuli were then degraded by low-pass spatial frequency filtering. Four stimuli (Silhouettes 2, 6, 11, and 12 from Figure 1) were used. In this experiment, unlike Experiments 4 and 5, blocking was accomplished by averaging only over regions of $3 \times 3,5$ $\times 5$, and $7 \times 7$ pixels, respectively. (A $1 \times 1$ average - a control condition - was also included.) This reduction in the number of values of the averaging region was necessary to reduce the number of experimental conditionsa number that was increasing substantially as we added conditions to earlier experimental designs.

Low-pass spatial frequency filtering was then applied to these blocked stimuli. Nominal low-pass cutoff limits of $.44, .61, .96$, and 1.31 cycles/deg were used. (This expanded range - in comparison with the bandpass cutoff values of earlier experiments-was chosen in order to scan more fully the range of variability of this variable.) The control conditions included the four aircraft silhouettes with blocking, but no spatial frequency filtering.

The results of Experiment 6 are shown in Figure 9. Since 6 subjects participated for five hourly sessions, each point in this figure is the mean performance score based on approximately 900 trials. The results of this experiment display an intermediate outcome. The effect of the combined blocking and low-pass filtering transformations are nearly the same as the results of Experiment 2 as shown in Figure 5. Blocking, which has a minor effect 
alone, seemed to produce little additional effect when compared with low-pass filtering alone. The only exception to this general result was a slight effect for the .44 cycles/deg condition, a condition that was not used in Experiment 2.

\section{Experiment 7: Combined Effect of Blocking and Low-Pass Filter-Reverse Order}

Experiment 7 was identical in all regards to Experiment 6 with one exception. The order of blocking and spatial frequency filtering was reversed. In this case, the stimuli were first low-pass spatial frequency filtered and then blocked. All other conditions of the two experiments were the same. In this case, the effect of this sequence of degradations was to produce blocks (and their accompanying high-frequency edges) in some of the conditions that would have been blurred if the degradations had been carried out in the reverse order. In other conditions, in which block size was small, the filtered and then blocked stimuli retained their blurred appearance.

The results of the experiment are shown in Figure 10. Since 8 subjects each participated in five daily sessions, each point on this figure represents the mean performance score based on 400 trials. The results of this experiment were approximately the same as in Experiment 6 . There was a modest effect of the low-pass filtering operation that is nearly identical to that found in Experiment 2. The blocking added little to the effect of the low-pass filter - merely a slight decrease in performance from one block size to the next larger one. Differences in the shapes of the curves in Figure 10 are minor, with much of the drop in performance occurring at higher spatial frequency cutoff values than in Figure 9. An important suggestion begins to emerge from the data shown in Figures 9 and 10: the order of the degradations seems to have little effect on discriminability.

\section{Experiment 8: Combined Effect of Blocking, Low-Pass Filter, and Visual Interference}

Experiments 8 and 9 are the ultimate combination experiments carried out in the present study. In these two

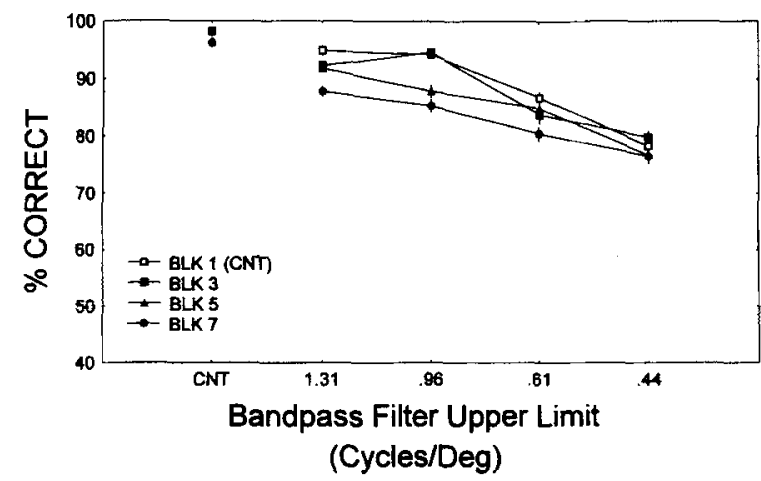

Figure 10. The results of Experiment 7, in which the blocking and the spatial frequency filtering experiments were carried out in sequential order, but in reverse order from that in Experiment 6 (filtering first, then blocking).

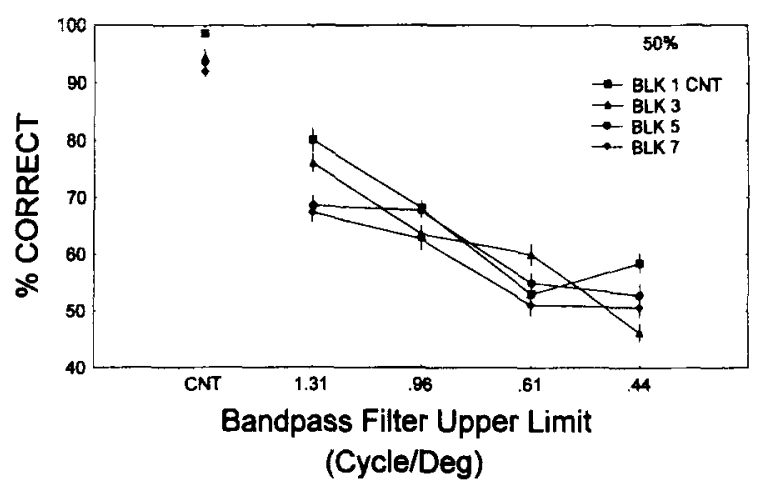

Figure 11. The results of Experiment 8, in which all three degradations - blocking, filtering, and visual interference-were combined. In this experiment, blocking was carried out first and then filtering. This figure shows the results for the $50 \%$ visual interference density. The controls in this case are for unblocked and unfiltered versions of the aircraft silhouettes that were utilized.

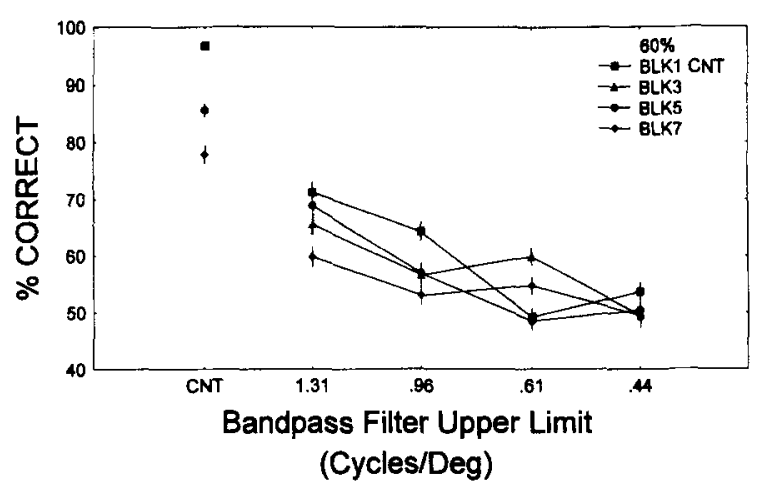

Figure 12. The results of Experiment 8 for the $60 \%$ visual interference density.

experiments, we combined both of the degrading transforms of primary interest-blocking and spatial frequency filtering - with random visual interference. The difference between Experiments 8 and 9 was simply the order in which the blocking and the spatial frequency filtering transforms were applied. In Experiment 8, blocking was carried out first, and the resulting blocked images were then spatial frequency filtered. In Experiment 9, the order of the two transformations was reversed. Four conditions of blocking were used by averaging over $1 \times 1$ (control condition), $3 \times 3,5 \times 5$, and $7 \times 7$ pixels. Four nominal spatial frequency cutoff limits of $.44, .61, .96$, and 1.31 cycles/deg were used to set the low-pass filtering operation in this case. Two levels of visual interference- $50 \%$ and $60 \%$-were used as challenges. Four control stimuli were also inserted into the experimental protocol. These control stimuli were blocked, but unfiltered, versions of the four aircraft silhouette stimuli (Silhouettes 2, 6, 11, and 12 from Figure 1) that were used in this experiment.

Figures 11 and 12 show the results of Experiment 8 for the two visual interference levels- $50 \%$ and $60 \%$, re- 


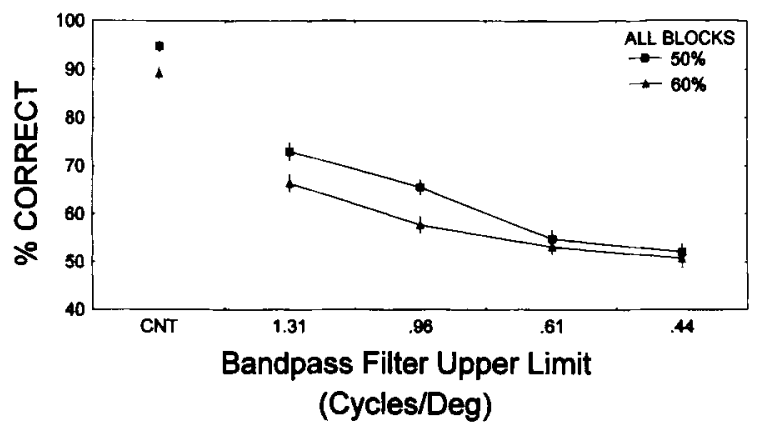

Figure 13. The results of Experiment 8 pooled across all blocking sizes and plotted for the $50 \%$ and $60 \%$ visual interference densities.

spectively (with the four blocking levels as a parameter) - as a function of the upper limit of the low-pass filter. Since 7 subjects participated for each of five daily sessions, each point on each of these two figures is the mean performance score based on 175 trials. Both of these curves display the minor differential effect of blocking (i.e., substantial overlap of the curves for the different blocking levels) on the discrimination of stimuli, but the stronger effect of spatial frequency filtering (i.e., a near constant decline as a function of the cutoff frequency).

In Figure 13, the same data have been plotted for the two levels of visual interference used, but pooled across all blocking levels. Each point is the mean performance score based on 700 trials. There is an obvious difference between the two curves, showing the regular effect of visual interference in this experiment. The two curves, however, do tend to overlap at the lower cutoff frequencies; this is probably a floor effect.

\section{Experiment 9: Combined Effect of Blocking, Low-pass Filter, and Visual Interference- Reversed Order}

Experiment 9 is identical in all regards to Experiment 8 , with two exceptions. First and most important, the order of the two degradation processes-blocking and spatial frequency filtering - was reversed. In this experiment, the spatial frequency filtering was carried out first and then the filtered stimuli were blocked. Second, three (rather than two) levels of visual interference $(40 \%, 50 \%$, and $60 \%)$ were used to more fully cover the response range because of the unexpectedly strong effect of the $60 \%$ visual interference level obtained in Experiment 8 at the lower cutoff limits.

The results of Experiment 9 are plotted in Figures 14, 15 , and 16 for the three visual interference levels. The data are separated in each figure on the basis of the size of the block over which the averaging was carried out following the spatial filtering. The results are plotted as a function of the upper limit of the low-pass filter expressed in cycles per degree of visual angle. Since 7 subjects participated on each of 4 days in this experiment, each point on each of these three figures is the mean performance score based on approximately 125 trials.
The results are pooled across the three blocking levels and replotted in Figure 17 to show the effects of the spatial frequency filtering across all blocking levels. In this figure, each plotted point is the mean performance score based on approximately 370 trials. The general trend of these data is very much the same as those in the previous experiment-a modest effect of blocking, but a stronger effect of the nominal upper frequency cutoff limit. However, as we shall shortly show, the discrimination scores for either order are virtually identical.

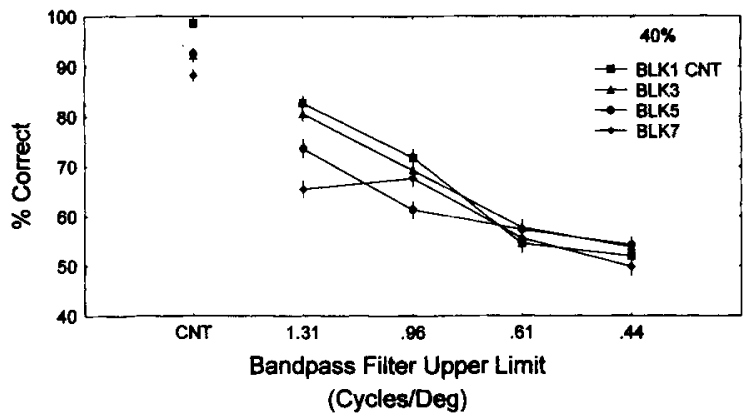

Figure 14. The results of Experiment 9, in which all three degradations - blocking, filtering, and visual interference-were combined, but in a reversed order from that in Experiment 8. In this experiment, spatial frequency filtering was carried out first, and the stimuli were then degraded by the blocking transformation. This figure shows the results for the $\mathbf{4 0} \%$ visual interference density.

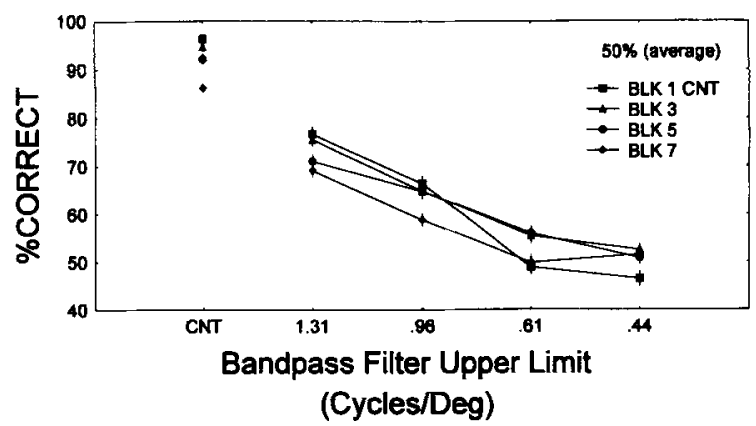

Figure 15. The results of Experiment 9 for the $50 \%$ visual interference density.

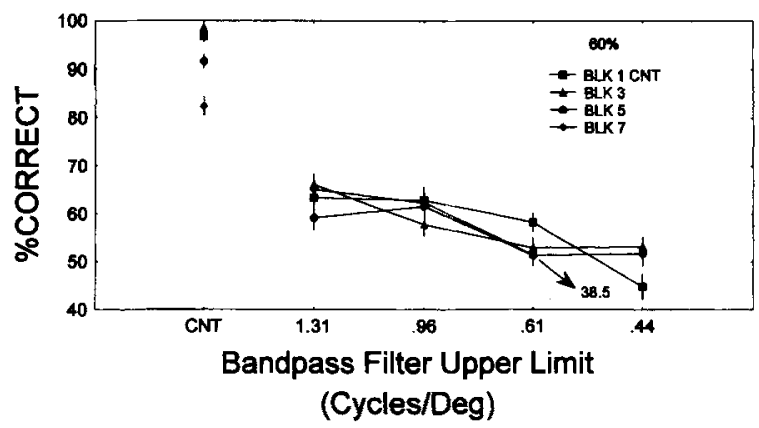

Figure 16. The results of Experiment 9 for the $60 \%$ visual interference density. 


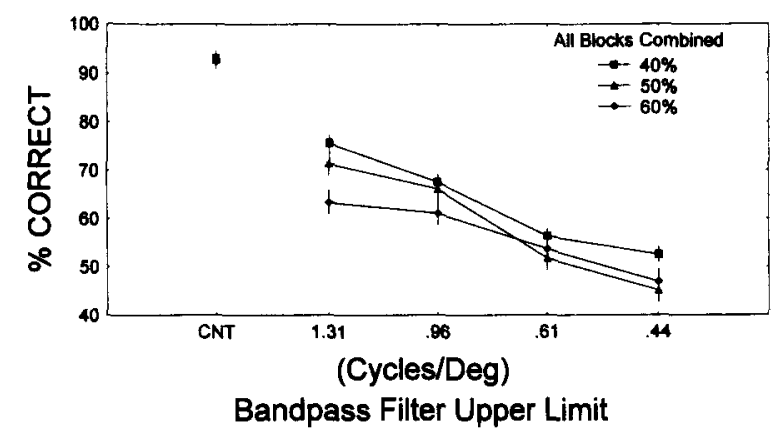

Figure 17. The results of Experiment 9 pooled across all blocking sizes for the three visual interference densities.

\section{DISCUSSION}

In this article, we have begun to describe some of the effects of combining three different stimulus degradations-blocking, spatial frequency filtering, and random visual interference. In brief summary, we have obtained the following main results:

1. The order in which the degradations are applied seems to be of no consequence. The analogue of multiplication represented by sequential transformation is, therefore, perceptually commutative.

2. Contrary to results obtained with a recognition task, sequential degradations with any combination of the three types of degradations always produced further decrements in performance. In no case was there any improvement of performance as degradations were cascaded.

Let us consider each of these results in turn.

\section{Discriminative Commutativity}

To better appreciate the data arguing for the discriminative commutativity that we have observed in this experiment, we compare the results of Experiments 3, 5, 8, and 9 in Figure 18. We have chosen the one common visual interference level $(60 \%)$ that was used in all four of these experiments for the purposes of this comparison. Clearly, the two curves from Experiments 8 and 9 essentially overlap. We also note, however, that the results of Experiment 3, in which only the spatial frequency degradation was applied, also overlap these two curves.

To further support the conclusion of commutativity of sequential degradations, in Figure 19 we have plotted the data for the $50 \%$ visual interference condition from Experiments 8 and 9. Since this level of visual interference was not used in Experiments 3 and 5, this figure is presented only to further authenticate the fact that the results of Experiments 8 and 9 are virtually identical at other levels of visual interference.

To further our argument for the discriminative commutativity for different orders of degradation, comparisons should also be made of the data displayed in Figures 9 and 10, results from experiments in which no visual interference was used, but in which blocking and spatial frequency filtering have been sequentially ap- plied in both orders. The data from these experiments also display very similar response levels. This comparison provides a third checkpoint for comparison and also supports the conclusion of a general discriminative commutativity for these two stimulus degradations.

The finding of discriminative commutativity at several levels of visual interference in the present experimental paradigm is contrary to the expectations that might have emerged if the situation had first been examined from two other points of view-their mathematical or graphic properties. First, examination of the graphic outputs of the two orders of degradation (as exemplified in Figure 20) makes it clear that the two orders produce very different results - the two images have distinctly different appearances, depending on the order in which the degradations are applied.

Each of these stimuli has been processed by exactly the same levels of blocking and spatial frequency filter-

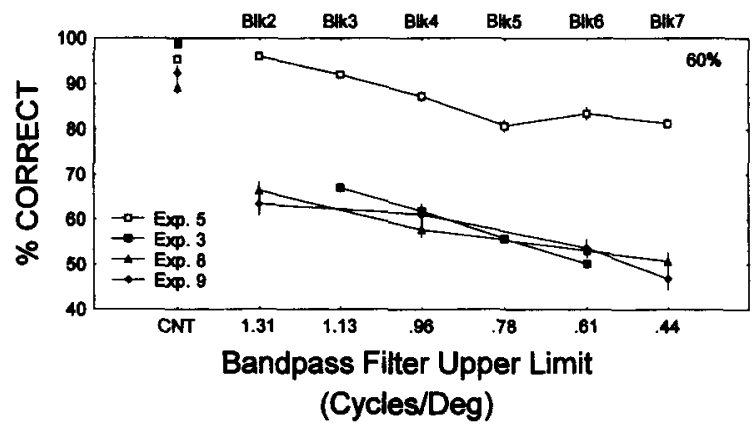

Figure 18. A comparison of the results of Experiments 3, 5, 8, and 9 for the $60 \%$ visual interference density. Experiment 5 examined blocking, Experiment 3 examined spatial frequency filtering, and Experiments 8 and 9 cascaded these two degradations in different orders. (Note that the horizontal axis for the blocked stimuli-Experiment 5 - is at the top of the figure and is scaled differently from the band-pass filter upper limit scale at the bottom of the figure.) This figure demonstrates that there is no evidence for an improvement in performance by cascading degradations in a discrimination task. It also demonstrates that the order in which blocking and spatial frequency filtering occurs is not significant - the degradations are commutative.

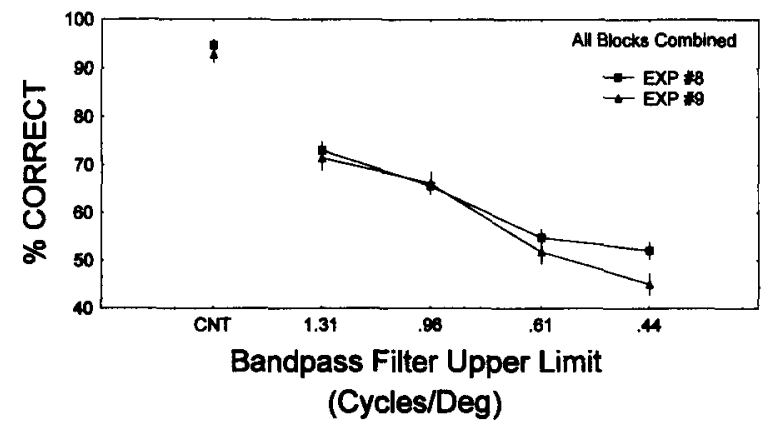

Figure 19. The results of Experiments 8 and 9 compared at the $50 \%$ visual interference density level, to show that the commutative effect also holds at lower visual interference densities than those compared in Figure 18. 
ing, but in reverse order. (For clarity, the challenging visual interference has not been added to these images.) Despite the large apparent difference between these two types of degraded stimuli, the two types are discriminated from others of their own type equally well, as shown in Figures 18 and 19.

The second source of what would have been an incorrect a priori expectation that the order of degradation would produce different psychophysical results comes from the mathematics of the situation. Unlike a simple convolution, which should be commutative, applying transforms such as spatial filtering and blocking should not be commutative. Specifically,

$$
h(g(f[x, y])) \neq g(h(f[x, y]))
$$

where $h$ and $g$ are the blocking and spatial frequency filtering transforms, respectively. This inequality is difficult to prove rigorously, and it may have some simple exceptions. Nevertheless, it is generally accepted by mathematicians that different orders of these two transformations will produce different results. The graphic "proof" shown in Figure 20 supports this argument. How very surprising, therefore, is the evidence for commutativity of these images in a discrimination task.

How, then, do we account for this discriminative commutativity? We suggest that it is mainly due to the fact that the high spatial frequencies are the attributes of the stimulus that are used in a discrimination task. In spite of the fact that the pictures look completely different when low-pass filtering and blocking are used in opposite orders, our results suggest that it is their common high spatial frequency (information) content that is mediating the discrimination task. The spatial frequency filtering operation removes the same high spatial frequency information from the stimulus, regardless of the order in which it applied. Thus, if the stimuli are first blocked, the high-frequency components of the original image influence the "blocked" shape. Upon low-pass spatial filtering, this critical information is removed. Similarly, if the targets are first low-pass filtered, the resulting "blobs" immediately lose the essential high spatial frequency information. When the "blobs" are subsequently blocked, the essential high spatial frequency information is already lost. In either case, the essential high-frequency information is not available for the discrimination task even though other irrelevant high frequencies may be introduced by the blocking procedure. The fundamental reason that the high spatial frequency information is important for discrimination is that discrimination is a process in which singular local discrepancies (local feature differences) can trigger a "different" response.

This outcome cannot be simply attributed to the fact that the blocking degradation has little effect under any condition. There are two indications that this is not the case. First, the experiments of the present study in which blocking alone was used do show decrements in performance as a function of the size of the block, especially when visual interference was present (see Figure 8). Thus, blocking is effective in situations in which the essential high-frequency information has not been removed by filtering. Blocking, therefore, becomes inconsequential when the essential high spatial frequency information is not present, regardless of the point in which it was removed.

Second, one only has to examine the large difference between the images shown in Figure 20 to appreciate that blocking does have a major role on the resulting image, depending upon the order. It is of special interest that these vastly different images are discriminated equally well.

This line of thought leads us to a solution to the problem of the difference between discrimination and recog-

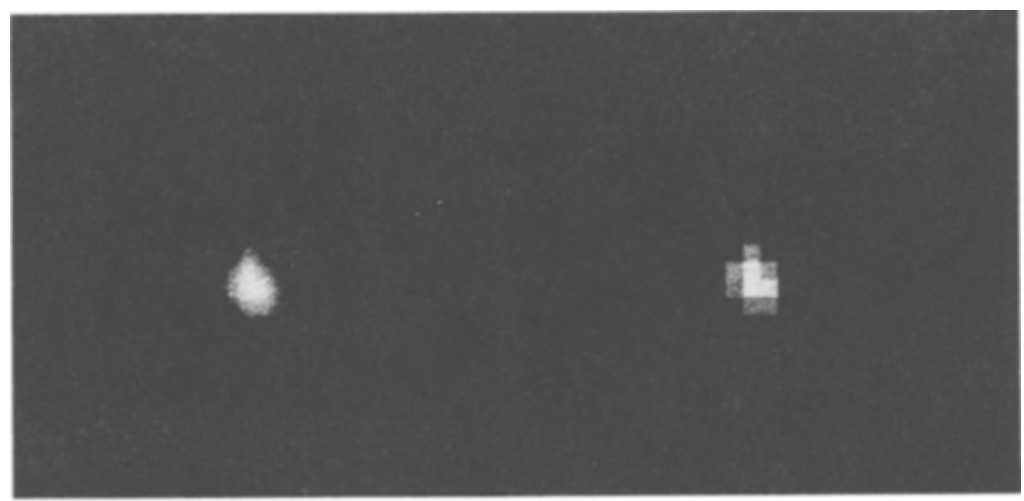

Figure 20. Two stimuli in which both blocking and spatial frequency filtering have been applied. (A) Blocking over a $7 \times 7$ averaging region first, followed by filtering with a nominal upper band-pass limit of 1.31 cycles/deg. (B) Filtering first, followed by blocking at the same values. 
nition. That solution is also framed in terms of the range of spatial frequencies used by recognition and discrimination, respectively.

\section{Discrimination Versus Recognition}

It is clear from the results obtained in this study that the use of a discrimination task, as opposed to a recognition task, produces a profoundly different result than that reported by Harmon and Julesz (1973). They reported improved "recognition" when blocked facial stimuli were subsequently spatial frequency filtered. They attributed this to the removal of bands of spatial frequencies "spectrally adjacent to the picture's spectrum" (p. 1194). In other words "sampled and quantized pictures may be more easily recognized after blurring the sampled and quantized picture" (p. 1194).

Our results show, to the contrary, that there is no improvement in a blocked image after it is low-pass spatial frequency filtered in a discrimination task. Spatial frequency filtering never improves the discrimination of blocked stimuli; performance levels are always equal to or lower than when the stimuli are processed by either degradation alone.

We now ask, why should this difference in results from the two tasks obtain? One possible answer to this question lies in the fact that the discrimination task requires much less information than does recognition. The discrimination task does not require specific identification with all of its attendant memorial and other cognitive processing, but only an answer to the following question: Are the two stimuli the same or different? Slight local discrepancies, insufficient to hamper recognition, may be sufficient to signal nonidentity. While there is considerable controversy in the literature concerning the amount of information and times required for detection, discrimination, and recognition, respectively, ${ }^{3}$ it is possible that in psychophysical studies of the kind reported here, this is simply a quantitative difference-progressively less information being required as one goes from recognition, to discrimination, to detection tasks.

However, our findings suggest a more specific and likely alternate explanation - that is, that discrimination and recognition are much less alike in terms of their fundamental information processing mechanisms than is usually assumed. Rather than simply being quantitatively different (in terms of, for example, the amount of information necessary), they may, in fact, be qualitatively different in terms of the kinds of information they utilize. Thus, whereas recognition may be mediated by the global, low spatial frequency information in an image, discrimination may be mediated by local, high spatial frequency information.

The data of the present experiment, therefore, support the conjecture that there is a qualitative difference between the mechanisms underlying recognition and discrimination. This qualitative difference is sufficient to produce complete disagreement between the basic phenomena observed in the two kinds of experiments. Thus, while recognition tasks produce the Harmon and Julesz
(1973) phenomenon, the phenomenon is not generalizable to all visual information processing tasks - specifically, as we have demonstrated here, discrimination. This conjecture is supported by the fact that the very same type of stimuli used in the present study will produce the Harmon and Julesz phenomenon when presented in a recognition task, an observation to be reported in the following article.

Finally, we want to emphasize that this is an experiment in shape discrimination. The spatial pattern of our stimuli, as degraded by the described operators, is the critical factor influencing discrimination. These spatial patterns are best quantified by the precise definitions of the stimuli and degradations that we have provided. We do not believe that the effects are explicable or adequately describable in terms of the kind of integrated measures used in computer vision studies for other purposes. Any single number that characterizes the state of the degraded image in comparison with the original image averages over the configuration and, therefore, obscures the key information concerning spatial relationships and components. For example, unified measures of contrast might have been used. However, our results are not likely to be explained by contrast alone. It is unlikely that there would be any substantial effect on discrimination if the level of degradation was held constant, but the contrast was varied over reasonably wide ranges. Similarly, if some measure of the difference between the degraded and undegraded images (e.g., the root mean square error) was used, stimuli that were quite different in their spatial configuration could have the same RMS deviation and yet produce completely different discrimination scores when compared with a standard. The point is that simple integrated measures, even though they may be correlated in some cases with the sequence of degradations, are not the salient, effective stimulus attributes that influence performance scores in this spatial pattern discrimination task. This is not to minimize their value in other kinds of information processing tasks, such as judging the lossy-ness of an image compression algorithm.

It should also be noted that whatever finally turns out to be the actual variable or variables determining our results, they are precisely defined by the way in which we applied the transforming degradations. Any potential measure or variable of interest can be recovered by applying the specified transforms to the specified stimuli. It is obviously impractical to tabulate all possible measures of the more than 400 distinctly different stimuli used in this study. When and if some simple measure of the stimulus is shown at some future time to be able to explain our results, the necessary information will be fully available in our explicit statement of the applied transformations.

The main point that we wish to make here is that global measures of stimulus energetics (or the relative energetics of different spatial frequency components) seem to us unlikely predictors of the results. At the present time, we do not have sufficient information to de- 
fine the most salient influences on shape perception, but we believe them to be spatial or configurational. A final theoretical statement depends on the results of this study, the companion article that follows, and, as indicated in that companion article, the results of other studies now underway. Given the suggestions mentioned in this article that Harmon and Julesz (1973) may have prematurely judged the theoretical context of their demonstration, we wish to avoid the same pitfall and not attempt at this time to develop a descriptive microtheory that cannot be generalized.

\section{REFERENCES}

BaChMANN, T. (1987). Different trends in perceptual microgenesis as a function of the spatial range of local brightness averaging. Psychological Research, 49, 107-111.

BaCHMANN, T. (1991). Identification of spatially quantized tachistoscopic images of faces: How many pixels does it take to carry identity? European Journal of Cognitive Psychology, 3, 87-103.

CAmprell, F. W., Howell, E. R., \& Johnstone, J. R. (1978). A comparison of threshold and suprathreshold appearance of gratings with components in the low and high spatial frequency range. Journal of Physiology, 284, 193-201.

Camprell, F. W., Howell, E. R., \& Robson, J. G. (1971). The appearance of gratings with and without the fundamental Fourier component. Journal of Physiology, 217, 17-18.

De Valors, R. L., \& DE Valois, K. K. (1988). Spatial vision. New York: Oxford University Press.

Durgin, F. H., \& ProfitT, D. R. (1993). Perceptual response to visual noise and display media (Final Report, NASA-NAG2-814). University of Virginia, Department of Psychology.

GINSBURG, A. P. (1978). Visual information processing based on spatial filters constrained by biological data (Report No. AMRL-TR78-129, Vols. 1-2). Wright-Patterson Air Force Base, OH: Aerospace Medical Research Laboratory.

GINSBURG, A. P. (1980). Specifying relevant spatial information for information evaluation and display design: An explanation of how we see certain objects. Proceedings of the SID, 21, 219-227.

Ginsburg, A. P., \& Evans, P. W. (1979). Predicting visual illusions from filtered images based upon biological data. Journal of the $O p$ tical Society of America, 96, 1443.

Graham, N. V. S. (1989). Visual pattern analyzers. New York: Oxford University Press.

Groner, R., Groner, M. T., Muller, P., Bischof, W. F., \& Di Lollo, V.
(1993). On the confounding effects of phosphor persistence in oscilloscopic displays. Vision Research, 33, 913-918.

HARMON, L. D., \& JUlesz, B. (1973, June 15). Masking in visual recognition: Effects of two-dimensional filtered noise. Science, 180, 1194-1197.

Hayes, T., Morrone, M. C., \& Burr, D. C. (1986). Recognition of positive and negative bandpass-filtered images. Perception, 15, 595602.

MARR, D. (1982). Vision. San Francisco: W. H. Freeman.

Morrone, M. C., Burr, D. C., \& Ross, J. (1983). Added noise restores recognizability of coarse quantized images. Nature, 305, 226-228.

PARKer, D. M., \& Costen, N. P. (1993). Spatial frequency and internal masking in face images. Perception, 22 (Suppl.), 48.

ShePherd, T., Utral, W. R., Dayanand, S., \& Lovell, R. (1992). A method for shift, rotation, and scale invariant pattern recognition using the form and arrangement of pattern-specific features. Pattern Recognition, 25, 343-356.

UTTAL, W. R. (1975). An autocorrelation theory of form detection. Hillsdale, NJ: Erlbaum.

UTTAL, W. R. (1983). Visual form detection in 3-dimensional space. Hillsdale, NJ: Erlbaum.

UTTAL, W. R. (1985). The detection of nonplanar surfaces in visual space. Hillsdale, NJ: Erlbaum.

UTTAL, W. R. (1987). The perception of dotted forms. Hillsdale, NJ: Erlbaum.

UTTAL, W. R. (1988). On seeing forms. Hillsdale, NJ: Erlbaum.

Uttal, W. R., Baruch, T., \& Allen L. (1994). Psychophysical foundations of a model of amplified night vision in target detection tasks. Human Factors, 36, 488-502.

Uttal, W. R., Baruch, T., \& Allen, L. (1995). Combining image degradations in a recognition task. Perception \& Psychophysics, 57, 682-691.

\section{NOTES}

1. The situation is not, however, as simple as it may at first seem. In Uttal et al. (1995), we will show that blocking followed by low-pass filtering and low-pass filtering followed by blocking both increase recognizability for the type of stimulus used in the present study!

2 . The automated Fourier frequency analyzer was developed by Charles Lunskis $\mathbf{J r}$., Thomas Shepherd, and Sriram Dayanand.

3. We are aware of the controversy surrounding the times necessary for discrimination and recognition, respectively. The reader is referred to Uttal (1988) for a complete discussion of this issue.

(Manuscript received January 14, 1994; revision accepted for publication December 16, 1994.) 